For Table of Contents use only

Structural Analysis of Dendrimers Based on Polyhedral Oligomeric Silsesquioxane and their Assemblies in Solution by Small-Angle Neutron Scattering: Fits to a Modified Two Correlation Lengths Model

Guangcui Yuan*, Xing Wang, Decheng Wu* and Boualem Hammouda*

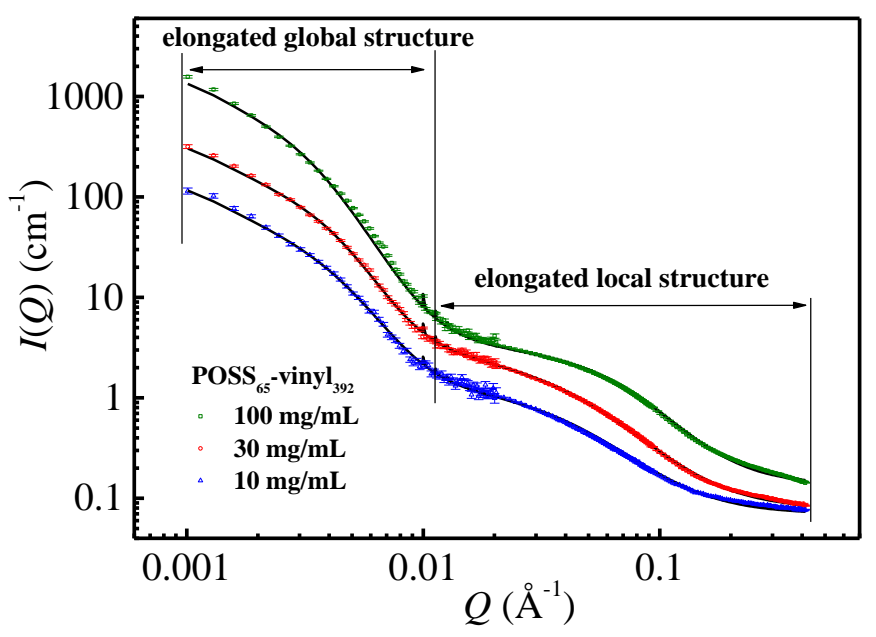

(C) 2016. This manuscript version is made available under the Elsevier user license http://www.elsevier.com/open-access/userlicense/1.0/ 


\title{
Structural Analysis of Dendrimers Based on Polyhedral Oligomeric Silsesquioxane and their Assemblies in Solution by Small-Angle Neutron Scattering: Fits to a Modified Two Correlation Lengths Model
}

\author{
Guangcui Yuan ${ }^{\mathrm{ab} *}$, Xing Wang $^{c}$, Decheng $W u^{\mathrm{c} *}$ and Boualem Hammouda ${ }^{\mathrm{a} *}$
}

${ }^{a}$ Center for Neutron Research, National Institute of Standards and Technology, Gaithersburg, Maryland 20899, USA

${ }^{b}$ Department of Polymer Engineering, The University of Akron, Akron, Ohio 443250, USA

${ }^{\mathrm{c}}$ Institute of Chemistry, Chinese Academy of Sciences, Beijing 100190, China

*Corresponding Authors:

Guangcui Yuan, 301-975-5098, guangcui.yuan@nist.gov

Boualem Hammouda, 301-975-3961, boualem.hammouda@nist.gov

Decheng Wu, 010-8261-1492, dcwu@iccas.ac.cn 


\begin{abstract}
The small-angle neutron scattering (SANS) technique has been used to investigate the structure of dendrimers based on polyhedral oligomeric silsesquioxane (POSS) in solution. Three generations and two distinct terminal groups (either vinyl or polyethylene glycol (PEG) terminal groups) as well as four concentrations for each dendrimer have been measured in deuterated tetrahydrofuran. Two main scattering regimes, one with a length scale of $\mathrm{nm}$ and the other with a length scale of sub-micron, are found to contribute to the SANS signals. In order to fit the SANS data, a new modified two correlation lengths model has been developed. Fit parameters include scale factors, correlation lengths, Porod exponents and "particle" elongation parameters. Our fit results show that the local structure does not correspond to single dendrimer molecules, but rather to POSS-rich domains surrounded by POSS-poor domains. The large-scale structure represents the overall morphology of multi-dendrimer aggregates. The flexible PEG outer links help to enhance the stability of inorganic POSS cages, and allow the hybrid polymers to adjust their conformation in order to respond to their surroundings. With increasing concentration, the local structure becomes more compact as evidenced by the decrease of correlation length and concurrent increase of the Porod exponent. The deformation of local structure during the compaction process is also observed. The structure becomes elongated at intermediate concentrations whereas it tends to be more globular at low and high concentrations.
\end{abstract}

Keywords: small-angle neutron scattering; polyhedral oligomeric silsesquioxane; modified two correlation lengths model 


\section{Introduction}

Polyhedral oligomeric silsesquioxane (POSS), a class of unique inorganic components with a definite nanostructure and ability to undergo polymerization, provides promising opportunities to construct novel hybrid organic/inorganic materials with advantageous properties. It has thus received significant research attention. A rich variety of well-defined POSS-containing hybrid polymers have been developed with different architectures, including telechelic polymers, ${ }^{1-3}$ starshaped polymers, ${ }^{4-6}$ block copolymers, ${ }^{7-9}$ and dendrimers. ${ }^{10-11}$ General reviews on the synthesis, functional properties, and applications of POSS derivatives can be found in the literature. ${ }^{12-16}$ The most investigated POSS species is octa-silsequioxane ( $\mathrm{T}_{8}$-POSS) which possesses a cubic inorganic $\mathrm{Si}_{8} \mathrm{O}_{12}$ core with side length of $5.3 \AA$ surrounded by eight tunable substituent groups. ${ }^{17-}$ 20

Owing to the inorganic constituent and rigid regular structure, POSS cages show strong aggregation propensity. Therefore, one particular focus is directed toward the higher-order structures of POSS derivatives in organic solvents (such as micellar and vesicular structures) or in the polymer matrix (such as lamellar and cylindrical structures). Many experimental 1, 7-10, 21-33 and theoretical ${ }^{34}$ investigations have demonstrated the aggregate morphologies of well-defined POSS-containing polymers and their response to processing parameters such as volume fraction and $\mathrm{pH}$. However, each specific POSS-containing hybrid polymer can behave very differently due to varying architectures, nature of organic periphery, media, etc. For example, Zhang et al. found that tadpole-shaped amphiphilic POSS-Poly( $N$-isopropylacrylamide) (PNIPAM) in aqueous solution forms core-shell nanostructured micelles with POSS molecules as the core and PNIPAM chains in the shell, ${ }^{26}$ while tadpole-shaped amphiphilic POSS-Poly(acrylic acid) (PAA) in aqueous solution does not self-assemble into typical core-shell micelles but rather into large aggregates where the POSS moieties are dispersed in the particles. ${ }^{27}$ They also found that, amphiphilic dumbbell-shaped POSS-functional PAA (POSS-PAA-POSS) forms ellipsoidal aggregates with sub-aggregates or crystals of POSS embedded in the PAA matrix. ${ }^{28}$

Overview of the past research on the structures of POSS-containing hybrid polymers formed in solution is included here. Transmission electron microscopy, scanning electron microscopy and atomic force microscopy are the most used techniques to visualize the morphology of higher order structure. However, due to the limitation on instrumental resolution, information about the 
details of the interior structure such as the packing structure of POSS nanocages (inside the large aggregates) and the chain conformation of the polymer constituents is missing. This shortage is severe especially for structures without periodicities, which is the typical case for structures formed in solution. In addition, the captured information by microscopy may not reflect the actual structure in solution because of the structural distortion during dehydration in sample preparation process. Moreover, light scattering is one of the most convenient solution characterization techniques to study the actual morphology. To obtain the shape information, the analysis of light scattering data generally relies on a dimensionless parameter $\rho$, the ratio between the radius of gyration and the hydrodynamic radius. Tan and He et al. ${ }^{21-24}$ used light scattering (static and dynamic) techniques to explore the physical properties and micellization behaviors of various POSS-based hybrid polymers. By comparing the experimental $\rho$ parameter with that of the theoretically perfect model and also with the support of other visualization techniques, they have identified multiple structures: a loosely packed multimolecular aggregate structure with $\rho<0.78$ in solutions of POSS/poly(lactide) hybrid star polymers, ${ }^{21}$ a vesicle or hollow-like structure with $\rho$ ranging from 1.10 to 1.25 in solutions of telechelic poly(styrene-ransodium styrenesulfonate) with POSS as end groups, ${ }^{22}$ and a core-corona micelle (a small POSS core surrounded by an extremely loose shell of long polymer chain) with large $\rho$ value ( $\rho=1.46)$ in solutions of amphiphilic multi-arm poly(ethylene oxide)/POSS polymer. ${ }^{23}$ However, it should be noted that since a light scattering method was employed in these studies, the understanding of spatial fluctuations of concentration were limited to the characteristic length scale of the wavelength of light. In the case with hierarchical structures, such as larger aggregates composed of sub-aggregates, light scattering is more sensitive to large aggregates, and the signal from unimolecules or sub-structures may be screened. To understand the hierarchical structure formed in solution, other scattering techniques (X-ray or neutron) which explore a smaller length scale are required.

To the best of our knowledge, there have been no reports on the use of small-angle neutron scattering (SANS) to investigate the structure of POSS-based macromolecules and their assemblies in solution. In this paper, we report SANS results from a series of POSS dendrimers in solution. These dendrimers are highly branched with eight branches at the central core and a multiplication factor of seven at each of the two generations. They were constructed from a $\mathrm{T}_{8}$ POSS core and an $\mathrm{AB}_{7}$ functional $\mathrm{T}_{8}$-POSS monomers, where the POSS moieties were 
embedded into the branches as the repeating units. Interestingly, these dendrimers form multidendrimer structures under certain concentration due to their physical interaction in solution. In a sense, each dendrimer could be considered as a sub-aggregate of POSS units linked by chemical bonds. So we will analyze how the local structure behaves inside the multi-dendrimer assemblies. By using one set of dendrimers with short vinyl terminal groups and the other set with long polyethylene glycol (PEG) terminal groups, the local POSS-rich domains can be differentiated, because the scattering length density of POSS units and of alkyl outer-links are quite different.

Usually, SANS data display many trends and shapes depending on the investigated microscopic structure and features. The data interpretation generally follows two ways, either using standard plots (such as Guinier or Porod plots) ${ }^{35}$ or using nonlinear least-squares fits to appropriate models. ${ }^{36}$ Standard plots give the first order interpretation of SANS data, and precise models give a more detailed approach at obtaining results. However, precise molecular models are not always available. An intermediate approach consists in using empirical models that reproduce the main trends observed in SANS data. In this work, we introduce a new modified two correlation lengths model which can be applied to scattering objects with two distinct sizes (here, the local POSS-rich domains and the global assembly in solution). The usefulness of this model and the structure analysis of the dendrimers and their assemblies are discussed based on the fit results.

\section{Experimental section}

\subsection{Materials}

The synthesis and characterization (including nuclear magnetic resonance spectroscopy, gelpermeation chromatography, dynamic light scattering etc.) of the dendrimers used in this work can be found in Wang et al.'s ${ }^{11}$ paper. Either vinyl or PEG chains with about 17 repeated ethylene glycol units are used as terminal groups of the dendrimers. The one- and two-generation (G1 and G2) dendrimers were synthesized in one and three steps respectively by construction from a functional $\mathrm{T}_{8}$-POSS core and an $\mathrm{AB}_{7}$ functional $\mathrm{T}_{8}$-POSS monomers. The PEG outerlinks were obtained by further substituting the vinyl outer-links with sulfhydryl ending PEG short chain (the details can be found in Supplementary Information). 
For the readers' convenience, the schematic diagram in Ref. 11 about the chemical structures of G0, G1 and G2 POSS dendrimers is reproduced in the current paper as Figure 1. Note that, in order to represent the hyperbranched structures, the schematic figure is not drawn to scale. The blue squares represent the POSS units and the green circles represent the terminal groups. The detail chemical structure of the dendrimers is provided in Supplementary Information. The G0 core possesses 1 POSS unit and 8 terminal groups, G1 dendrimer possess 9 POSS units and 56 terminal groups, G2 dendrimer possess 65 POSS units and 392 terminal groups. The side length of a cubic POSS unit is $5.3 \AA$, and the average length per ethylene glycol unit is $2.46 \AA{ }^{37}$ So when PEG is used as the terminal group, the length of each terminal group is much longer than each POSS unit, and also longer than the distance between two linked POSS units (12 chemical bonds on the main chain).

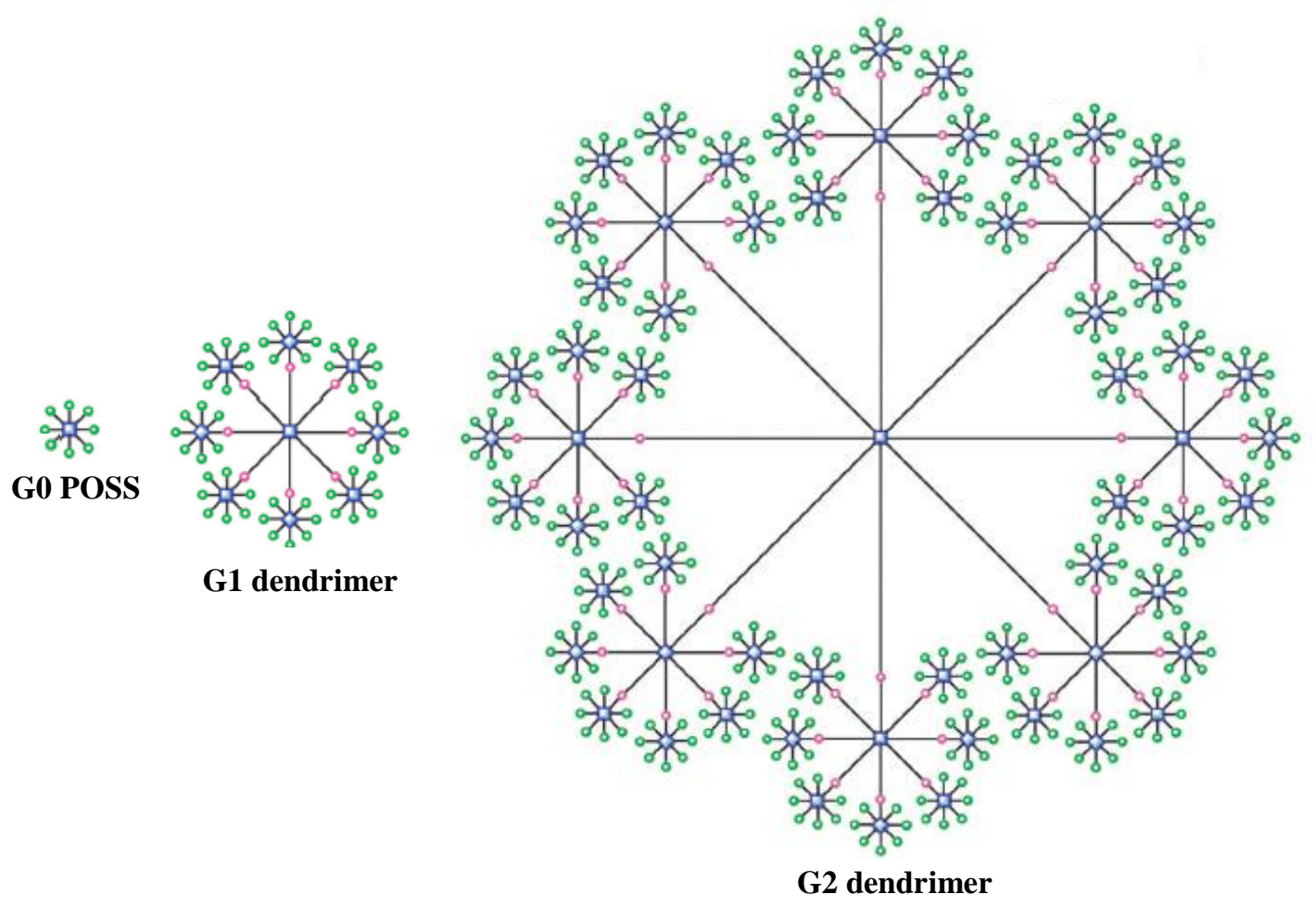

Figure 1. Schematic chemical structures of G0, G1 and G2 dendrimers. Reprinted from Ref. 11 with minor modification at one substitutional group of G0 POSS.

In short, two G0 dendrimers $\left(\mathrm{POSS}_{1}-\mathrm{Vinyl} \mathrm{l}_{8}\right.$ and $\left.\mathrm{POSS}_{1}-\mathrm{PEG}_{8}\right)$, two G1 dendrimers $\left(\mathrm{POSS}_{8}-\mathrm{Vinyl}_{56}\right.$ and $\mathrm{POSS}_{8}-\mathrm{PEG}_{56}$ ) and two G2 dendrimers $\left(\mathrm{POSS}_{65}-\mathrm{Vinyl}_{392}\right.$ and $\mathrm{POSS}_{65}-\mathrm{PEG}_{392}$ ) were used in this 
study, and each dendrimer was measured under four concentration conditions $(c=3,10,30,100$ $\mathrm{mg} / \mathrm{mL})$.

\subsection{Small-Angle Neutron Scattering Measurement}

SANS was performed to characterize the POSS dendrimers and their assemblies in deuterated THF solution. The NGB-30m SANS instrument at the National Institute of Standards and Technology Center for Neutron Research (NCNR) was used with standard configurations corresponding to low- $Q$, intermediate- $Q$ and high- $Q$ ranges. Here, $Q$ is the scattering variable. These correspond to sample-to-detector distances of $13 \mathrm{~m}, 4 \mathrm{~m}$ and $1 \mathrm{~m}$ respectively. The neutron wavelength was $6 \AA$ for the last two configurations and $8.4 \AA$ for the first one. The low$Q$ configuration made use of neutron focusing lenses in order to reach the lowest possible scattering variable $Q_{\min }$. The three configurations cover three orders of magnitude in scattering scale (from $Q=0.001 \AA^{-1}$ to $\left.Q=0.5 \AA^{-1}\right)$. All measurements were performed at ambient $\left(25{ }^{\circ} \mathrm{C}\right.$ ) temperature.

\section{The modified two correlation lengths model}

SANS data are described qualitatively at first with a focus on the overall trends. Typical trends are included in order to make observations. Figure 2 gives the absolute scattering intensity $I(Q)$ obtained from G2 dendrimers at various concentrations. In this and other figures, statistical error bars correspond to one standard deviation. First, it is clear that two main scattering regimes are observed (see data in Fig. 2a, samples with vinyl outer-links), one in the high- $Q$ regime with a length scale of $\mathrm{nm}$ and the other in the low- $Q$ regime with a length scale of sub-micron. Second,

there is no Guinier ${ }^{31}$ region for the data sets, meaning that the size (radius of gyration) of the characteristic structures cannot be determined. Correlation lengths will thus be a better choice to describe the size of the characteristic structures. Third, focus is put on the intermediate- $Q$ regime, it will be found that the scattering objects are elongated. This is deduced from the slope of the $\log (I)$ vs. $\log (Q)$ plot in the intermediate- $Q$ regime. A horizontal line (slope $=0$ ) would point to spherical objects, a line with slope $=1$ would point to cylindrical shape, while a line with slope between 0 and 1 points to elongated (ellipsoidal) objects. The elongation seems to 
change with concentrations. The trend of varying elongation with concentration is more evident in another G2 dendrimer with PEG outer-links (see data in Fig. 2b). A dashed line $I \sim 1 / Q^{0.75}$ in Fig. $2 b$ provides a guide to the eyes for the slope corresponding to the elongation parameter in intermediate- $Q$ regime. Fourth, the multi-dendrimer crowding becomes very pronounced as the concentration increases to $100 \mathrm{mg} / \mathrm{mL}$, so that the scattering from the highest fraction crosses over that for the lower fraction (green curve in Fig. 2b), and the correlation length becomes even smaller. Lastly, the high- $Q$ Porod exponents also change with concentration. As indicated by the dotted lines $I \sim 1 / Q^{2.0}$ and $I \sim 1 / Q^{3.5}$ in Fig. 2b, the high- $Q$ Porod exponents POSS $_{65}-\mathrm{PEG}_{392}$ point to a mass fractal (Porod exponents less than 3) at $3 \mathrm{mg} / \mathrm{mL}$ and to a surface fractal (Porod exponents close to 4) for $100 \mathrm{mg} / \mathrm{mL}$.

In order to reproduce the main common features of the scattering data, an empirical model for mass fractals is used. The scattering intensity is of the form:

$$
I(Q)=\frac{1}{Q^{s}} \frac{C}{1+(Q L)^{m}}+B
$$

This is a modified version of the correlation length model where an elongation feature $1 / Q^{s}$ has been added. The numerical value of exponent $s$ is between 0 and $1 . C$ is the scale factor and $m$ is the high- $Q$ Porod exponent. In the Gaussian chain case (i.e., with Porod exponent of $m=2$ ), the correlation length model function becomes a simple Lorentzian for which the correlation length $L$ represents a characteristic length scale of a mass fractal structure.

To include the low- $Q$ structure along with the high- $Q$ structure, a modified two correlation lengths model is used instead.

$$
I(Q)=\frac{1}{Q^{s_{1}}} \frac{C_{1}}{1+\left(Q L_{1}\right)^{m_{1}}}+\frac{1}{Q^{s_{2}}} \frac{C_{2}}{1+\left(Q L_{2}\right)^{m_{2}}}+B
$$

Index 1 is used for the low- $Q$ feature while index 2 is used for the high- $Q$ feature. A constant $(Q$ independent) background $B$ has been added to represent the incoherent scattering background at high- $Q$. 

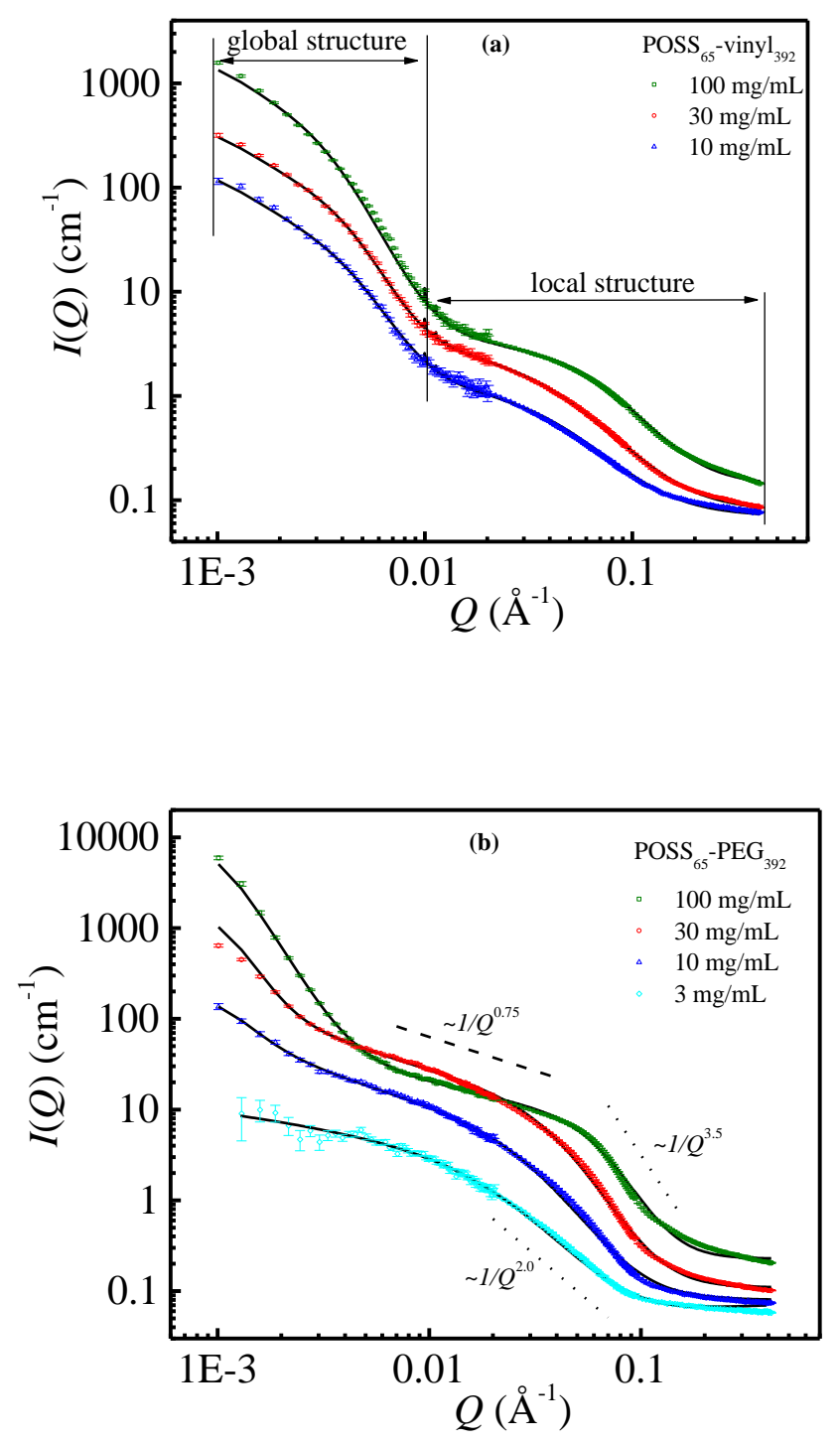

Figure 2. Experimental SANS data (symbols) and the corresponding fits (black solid lines) to the modified two correlation lengths model for the entire $Q$-range for G2 POSS dendrimers with vinyl outer-links (a) and PEG outer-links (b) at various concentrations.

A basic assumption of the modified two correlation lengths model is that two features (here one corresponds to the global aggregates and the other corresponds to the local structure, as will be discussed later) on the pair distance distribution function could be well decoupled. During the 
fitting, nonlinear least-squares fits are performed for the high- $Q$ SANS data first. A power law model $\left(A / Q^{n}\right)$ has been added in order to fit the upturn at low- $Q$ and a background term has also been included. Then, for data sets which include information of both the global aggregates and the local structure, a modified two correlation lengths model (equation 2) was applied by fixing the previous fit parameters for the high- $Q$ part. The black solid lines in Fig. 2 are the corresponding nonlinear least-squares fits to two modified correlation lengths model for the entire $Q$-range window for various samples. This model reproduces the actual data very well. The range of uncertainty in each fitted parameter was determined by fixing that parameter at various values and allowing the other parameters to vary during the fitting within physically reasonable limits. In this way the maximum uncertainty of the fitted parameters was found to be approximately $10 \%$.

As the fit parameters include scale factors, correlation lengths, Porod exponents and elongation parameters for both the low- $Q$ and high- $Q$ structures, nonlinear least-squares fits yielded a rich harvest of fitting parameters for the various samples. Besides, each specific dendrimer behaves differently in the experimental concentration range. However, some common features between various hybrid polymers, including the effects of generation, terminal group and concentration on the structure, are discussed in what follows.

\section{Data analysis and discussion}

\subsection{Low- $Q$ and high- $Q$ structures}

Since two main scattering regimes are observed, one would naturally think that the high- $Q$ information points to the single-dendrimer molecules while the low- $Q$ information points to dendrimer aggregates. We will discuss below, whether this is the case or not.

There is no doubt that the low- $Q$ structure is related to the multi-dendrimer aggregate. First of $a l l$, we have observed the gradual upturn of the low- $Q$ part in Fig. $2 \mathrm{~b}$, which indicates that the aggregation appears when the concentration reaches a certain value (critical aggregation concentration). Second, as shown in Figure 3, the characteristic correlation length of the low- $Q$ part $\left(L_{1}\right)$ is in the sub-micron scale, which is much larger than the size of single dendrimers. 
Wang et al. ${ }^{11}$ have determined the size of the isolated dendrimers which exist in very dilute solution using various methods. Their dynamic light scattering data revealed that the hydrodynamic diameters for dispersive $\mathrm{POSS}_{9}-\mathrm{Vinyl}_{56}$ and $\mathrm{POSS}_{65}-\mathrm{Vinyl}_{392}$ are $74 \pm 2$ and $188 \pm$ $4 \AA$, respectively.

Meanwhile, as shown in Figure 3, the characteristic correlation length of the high- $Q$ part $\left(L_{2}\right)$, (around $11 \AA$ for $\mathrm{POSS}_{9}$-Vinyl 56 and $22 \AA$ for $\mathrm{POSS}_{65}-$ Vinyl $_{392}$ ) are much less than the size of the corresponding single dendrimers. The striking difference in length scale rules out the idea that we are observing the structure of single-dendrimer molecules at high- $Q$ for dendrimers with vinyl outer-links. Furthermore, these small length scales are independent of the length of the outer-links (vinyl or PEG). The $L_{2}$ values obtained from dendrimers with PEG outer-links at 100 $\mathrm{mg} / \mathrm{mL}$, i.e., $10 \AA$ for $\mathrm{G} 1$ dendrimer and $18 \AA$ for $\mathrm{G} 2$ dendrimer (data shown in Figure 5a, the concentration effect will be discussed later), are nearly the same as for dendrimers with vinyl outer-links under fitting uncertainty. For local scales, the POSS units should stay constant and similar with the generation number. Therefore, the small length scale and the independence on the outer-links lead us to the conclusion that the high- $Q$ information points to the intra-dendrimer local structure, instead of free dendrimer molecule. That is, the SANS technique uncovered an inhomogeneous morphology consisting of POSS-rich and POSS-poor regions inside the dendrimer. The intra-dendrimer localization phenomenon, which originates from the strong aggregation propensity of POSS units and is strongly related to the branched structure of the dendrimer, will lead to global aggregates between dendrimers. Or conversely, the assembly between dendrimers must be accompanied by the deformation of single dendrimer and the local structural re-arrangement.

Before moving forward, two points about the correlation length must be addressed. First, according to the modified two correlation lengths model, the correlation length is not necessarily the actual size of a real object, but gives an idea about the length scale relating to density/concentration fluctuations. For example, in semidilute polymer solutions, the correlation length represents the size of a "mesh" or "blob" composing polymer chains, or represents the range of the spatial correlation of concentration fluctuation in the system (distance between entanglement points). Second, we do not postulate that the scattering behavior is governed by the POSS units, because the scattering intensity is contributed not only from the contrast between the 
inorganic POSS units and d-THF, the contrast between the organic constituents (like vinyl, PEG) and d-THF, but also from the contrast between the POSS units and organic component. Here is some neutron scattering length densities for reference: $6.35 \times 10^{-6} \AA^{-2}$ for deuterated THF, $0.69 \times$ $10^{-6} \AA^{-2}$ for PEG bulk, and $1.57 \times 10^{-6} \AA^{-2}$ for octasiloxane (vinyl-POSS). Since the driving force for aggregation is mainly due to the inorganic constituent and rigid regular structure of POSS units, it is fair to say that an inhomogeneous morphology consisting of inorganic constituent POSS-rich and POSS-poor regions within the dendrimers is uncovered. Accordingly, there should be organic constituent polymer-rich and polymer-poor regions which may be flexible and permeable.

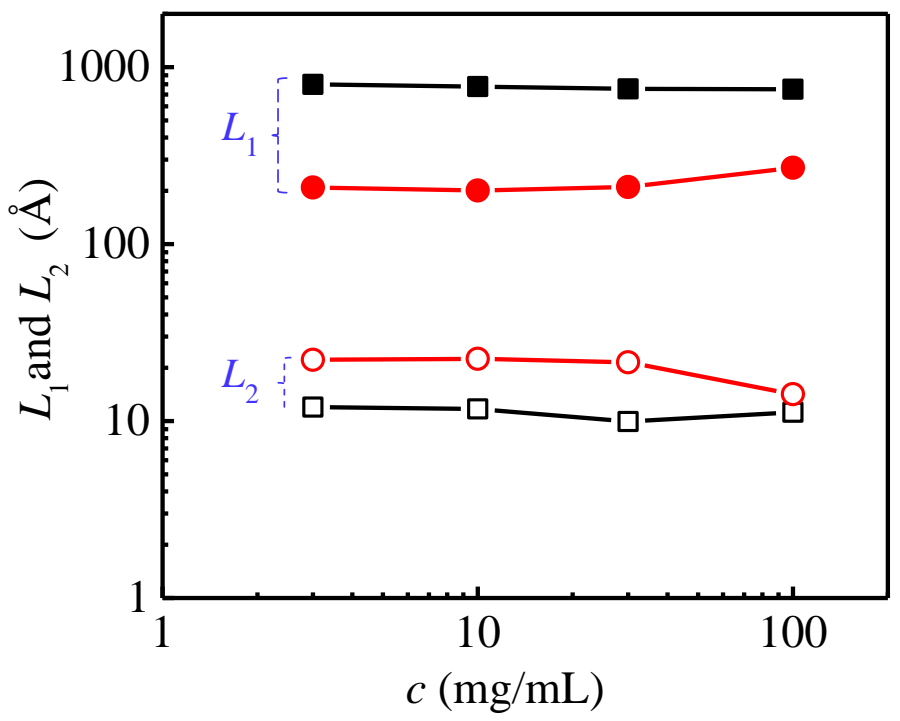

Figure 3. Variation of two correlation lengths $\left(L_{1}\right.$ and $\left.L_{2}\right)$ with dendrimer concentration for the two (low- $Q$ and high- $Q$ ) scattering modes. $L_{1}$ is in the sub-micron scale, and $L_{2}$ is in the nanometer scale. Black symbols are for $\mathrm{POSS}_{9}-\mathrm{Vinyl}_{56}$ and red symbols are for $\mathrm{POSS}_{65}-\mathrm{Vinyl}_{392}$.

This corollary that the nanometer correlation length corresponds to local structure while the sub-micron correlation length corresponds to global aggregate structure is reasonable. The POSS dendrimers embed more than one POSS units inside each dendrimer. On the intra-dendrimer scale, the POSS units can pack locally to form small domains within dendrimers; on the interdendrimer scale, assembly between dendrimers forms global aggregates. It is known that the side 
length of each cubic POSS cage is $5.3 \AA$. Thus the experimental $L_{2}$ values (i.e. $11 \AA$ for $\mathrm{POSS}_{9}$ Vinyl $_{56}$ and $22 \AA$ for POSS $_{65}-$ Vinyl $_{392}$ ), which are about two and four times of the side length for G1 dendrimer and for G2 dendrimer respectively, might indicate that every two (for G1) or four (for G2) adjacent rigid POSS cages closely pack inside the dendrimers and become a local POSS-rich domain detected by SANS. This non-uniform distribution picture is consistent with the computational models from the quantum chemical method and molecular mechanic calculation for the dendrimers. ${ }^{11}$

\subsection{The role of organic constituents}

The localization of POSS domains are affected by the organics constituents. Even for dendrimers with vinyl terminal groups, the organic constituents account for a considerable proportion $\left(\approx 30 \%\right.$ mass fraction) to the molecular weight. In Fig. 3 , the $L_{2}$ for higher generations which embed more POSS units inside the limited space of a dendrimer show larger values (red symbols) than lower generation ones (black symbols), but the $L_{1}$ values for higher generation dendrimers (red symbols) are smaller than for lower generation dendrimers (black symbols). The reason may be due to the periphery structure of low generation dendrimers that are more open, which allows other dendrimers to accumulate more. The higher generation dendrimers have denser surface organic groups, which helps to improve the infiltration performance, and thus lead to smaller aggregates.

Introduction of large PEG terminal groups helps us to explore the effect of the organic constituents on local packing, as it has more remarkable effect. At least, the role of steric hindrance should not be ignored. PEG has good solubility in THF. It is expected that grafted PEG blocks are able to enhance the stability of POSS cages. Indeed, a single POSS core surrounded by eight substituent PEG short chains is stable in solution, even when the concentration of G0 POSS reaches $100 \mathrm{mg} / \mathrm{mL}$. Figure 4 shows the stability contrast between $\mathrm{POSS}_{1}-\mathrm{Vinyl}_{8}$ and $\mathrm{POSS}_{1}-\mathrm{PEG}_{8}$. At the same concentration, there is a steep upturn for $\mathrm{POSS}_{1^{-}}$ Vinyl $_{8}$ at low- $Q$ indicating strong aggregation, while the data points are leveling-off at low- $Q$ for $\mathrm{POSS}_{1}-\mathrm{PEG}_{8}$ which means that $\mathrm{POSS}_{1}-\mathrm{PEG}_{8}$ is well dispersed. It is clear that PEG terminal groups help to inhibit the aggregation. 


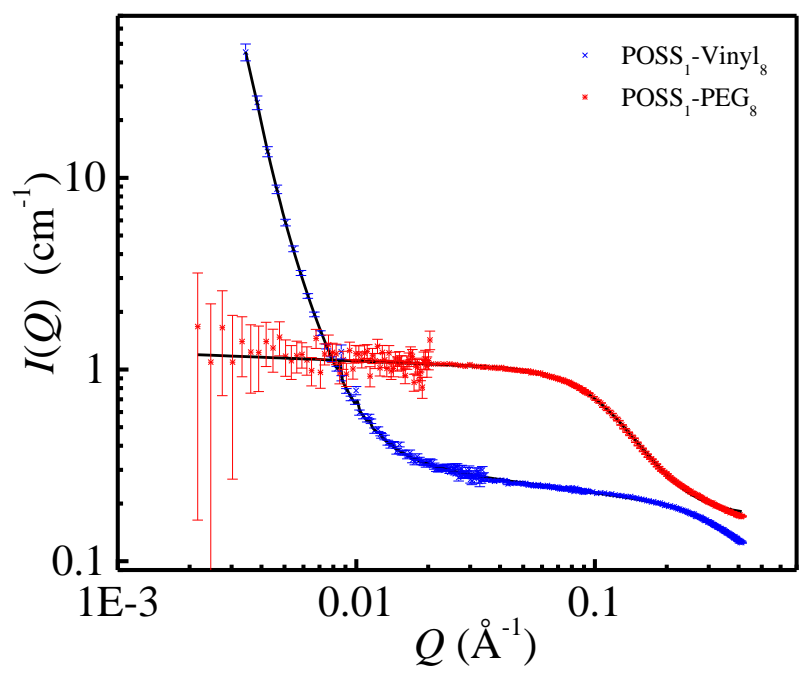

Figure 4. Experimental SANS data (symbols) for $\mathrm{POSS}_{1}-\mathrm{Vinyl}_{8}$ and $\mathrm{POSS}_{1}-\mathrm{PEG}_{8}$ at concentration of $100 \mathrm{mg} / \mathrm{mL}$. The black solid lines are corresponding fits to the modified two correlation lengths model for the entire $Q$-range.

However, end group substituents cannot completely eliminate the strong aggregation propensity between, and inside dendrimers. Multi-dendrimer aggregates appear as the environment gets crowded with increasing concentration (see data in Fig. 2b). Note that we are observing only the tail of the low- $Q$ feature and that we do not have the Guinier region for that feature. So here our focus is put on the intra-dendrimer local crowding. The trend shown in Figure 5a, concerning the variation of $L_{2}$ values with increasing concentration for $G 1$ and $G 2$ dendrimers with PEG outer-links, is apparently different from what we see in Fig. 3 for dendrimers with vinyl outer-links. In Fig. $3, L_{2}$ is almost a constant with increasing concentration. Due to the small vinyl substituent groups, the POSS cages have been closely packed at relatively low concentration and cannot get closer even when the external environment becomes crowded. In Fig. 5a, $L_{2}$ dramatically drops with increasing concentration and finally reaches the same value of that with vinyl outer-links at $100 \mathrm{mg} / \mathrm{mL}$. For local scales, the POSS units should stay constant and similar with the generation number, but polymer chains can contract and lead to different sizes on a local scale. At low concentration, the expanded PEG blocks separate the POSS domains within dendrimers resulting in a larger $L_{2}$ value. As the 
environment becomes crowed, the flexible PEG blocks overlap and the rigid POSS cages are forced to get closer.

\subsection{The Porod exponents}

The decrease in $L_{2}$ is companied by a change in the packing structure of the local domains as it should, because the dendrimer branches get crowded as the fraction increases. This trend of compaction can be inferred from the increasing of the Porod exponents (noted $m_{2}$ ) shown in Figure 5b. The closer the packing is, the larger $m_{2}$ gets. For reference, a Porod exponent $m=4$ corresponds to particles with smooth surfaces, while $m=3$ corresponds to ones with very rough surfaces or collapsed polymer chains in bad solvent. An exponent $m=2$ can represent scattering either from Gaussian polymer chains or from a two-dimensional structure, and $m=1$ represents scattering from a stiff rod. ${ }^{38}$ A Porod exponent less than 3 is called mass fractal, and a Porod exponent close to 4 is called surface fractal. Therefore, the $m_{2}$ for G2 dendrimers are normally larger than for G1 dendrimers because it is much more crowded inside the G2 dendrimers. In Fig. 5b, we also find that at the highest experimental concentration ( $c=100 \mathrm{mg} / \mathrm{mL})$, the local domain is no longer a mass fractal, but becomes a surface fractal.

The dendrimers with vinyl outer links lose much of their ability to adjust their conformation as concentration increases because of the less flexible organic components. So the $m_{2}$ data for dendrimers with vinyl outer-links, similarly to their corresponding $L_{2}$ in Fig. 3, does not change much as concentration increases. Data are not shown here. 

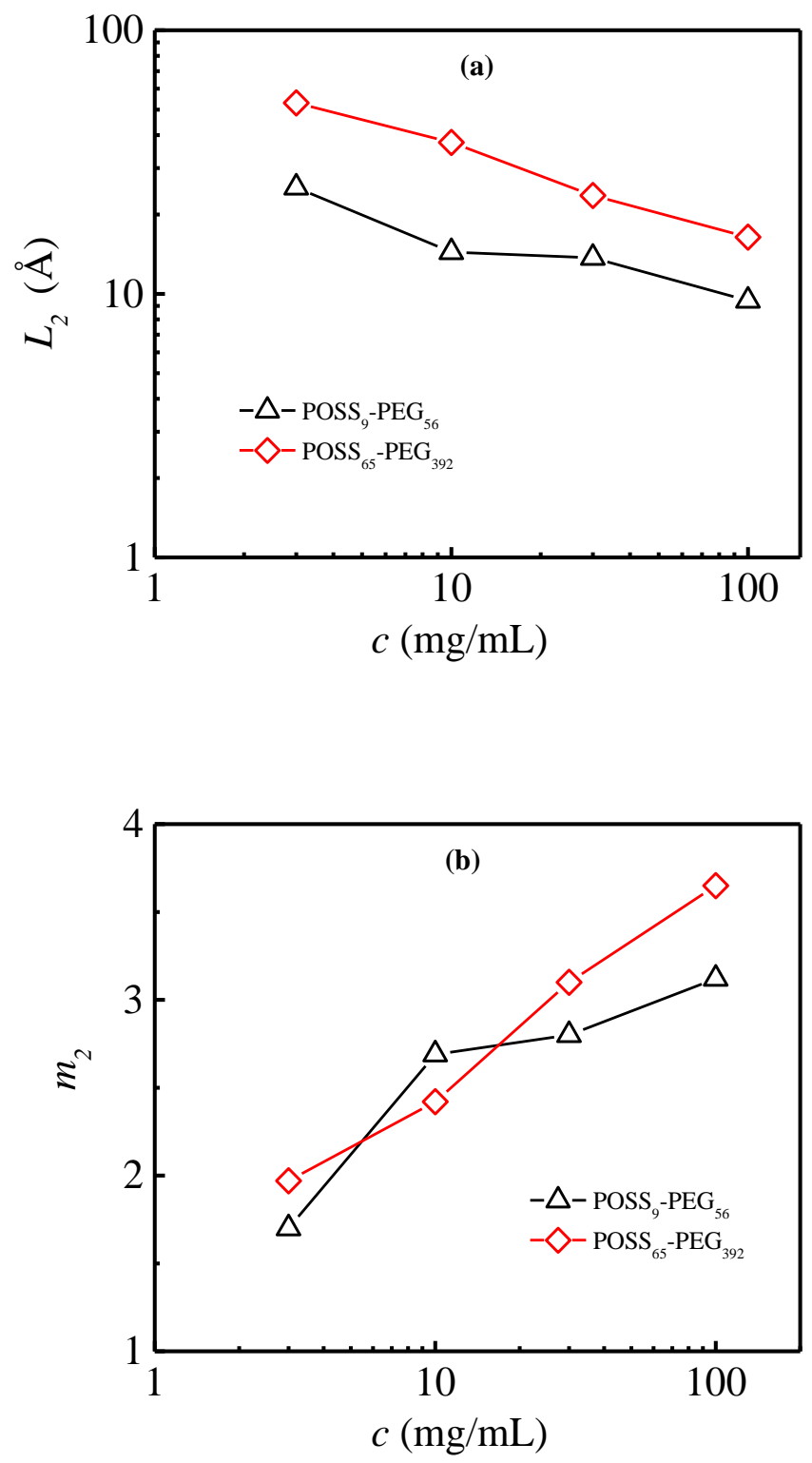

Figure 5. Variation of (a) correlation length, $L_{2}$, and (b) Porod exponent, $m_{2}$, with increasing concentration for the $G 1$ and $G 2$ dendrimers containing PEG terminal groups.

\subsection{Elongation}

A striking phenomenon found in this study is that the local domain is elongated and changes with concentration. An example has been shown in Fig. $2 b$ for $\mathrm{POSS}_{65}-\mathrm{PEG}_{392}$. At low mass 
fraction and at high mass fraction, the local structures tend to be globular, while at intermediate mass fractions, dendrimers form more elongated structures. This trend reversal as the fraction is increased can be also found in other generations, and is more evident in dendrimers with PEG outer-links. Another remarkable example shown here is for $\mathrm{POSS}_{1}-\mathrm{PEG}_{8}$ (see data in Figure 6a). $\mathrm{POSS}_{1}-\mathrm{PEG}_{8}$ is of globular shape at low concentration $(3 \mathrm{mg} / \mathrm{mL})$ as it should be since it possesses one POSS central cage symmetrically surrounded by 8 PEG terminal groups. As the concentration is increased to $10 \mathrm{mg} / \mathrm{mL}$, the molecule becomes elongated. As the concentration is increased further, there is no evidence for elongation, and structures appear globular again due to crowding. For $\mathrm{POSS}_{1}-\mathrm{PEG}_{8}$, the stretching may be due to the excluded volume effect, that is, the expanded PEG blocks need to overcome a potential barrier before they overlap. For higher generation dendrimers, when POSS units aggregate locally to form small domains within dendrimers, they involve stretching of PEG that are linked to them, and vice versa. This produces the observed elongation or stretching bias. An elongation parameter $s_{2}$ is included in the fit model and represents the data very well. Figure $6 \mathrm{~b}$ shows the variation of $s_{2}$ with increasing concentration for different generation dendrimers with PEG outer-links.

The fitting shows that the elongation parameter $s_{1}$ is approximately 1 for most global aggregates, representing ellipsoidal or cylindrical objects. The picture in real space might somehow look like the model proposed by Zhang et $a l .^{28}$, which presents ellipsoidal local domains distributed inside global ellipsoids. Their model is mostly based on transmission electron microscopy images of self-assembled aggregates of POSS-PAA-POSS. Although the molecular architecture of the dendrimers studied here is significantly different from Zhang's dumbbell shaped molecules, the morphologies of this kind of hybrid polymers are dictated by similar interactions and thermodynamics of mixing/demixing, which may lead to similar structure. 

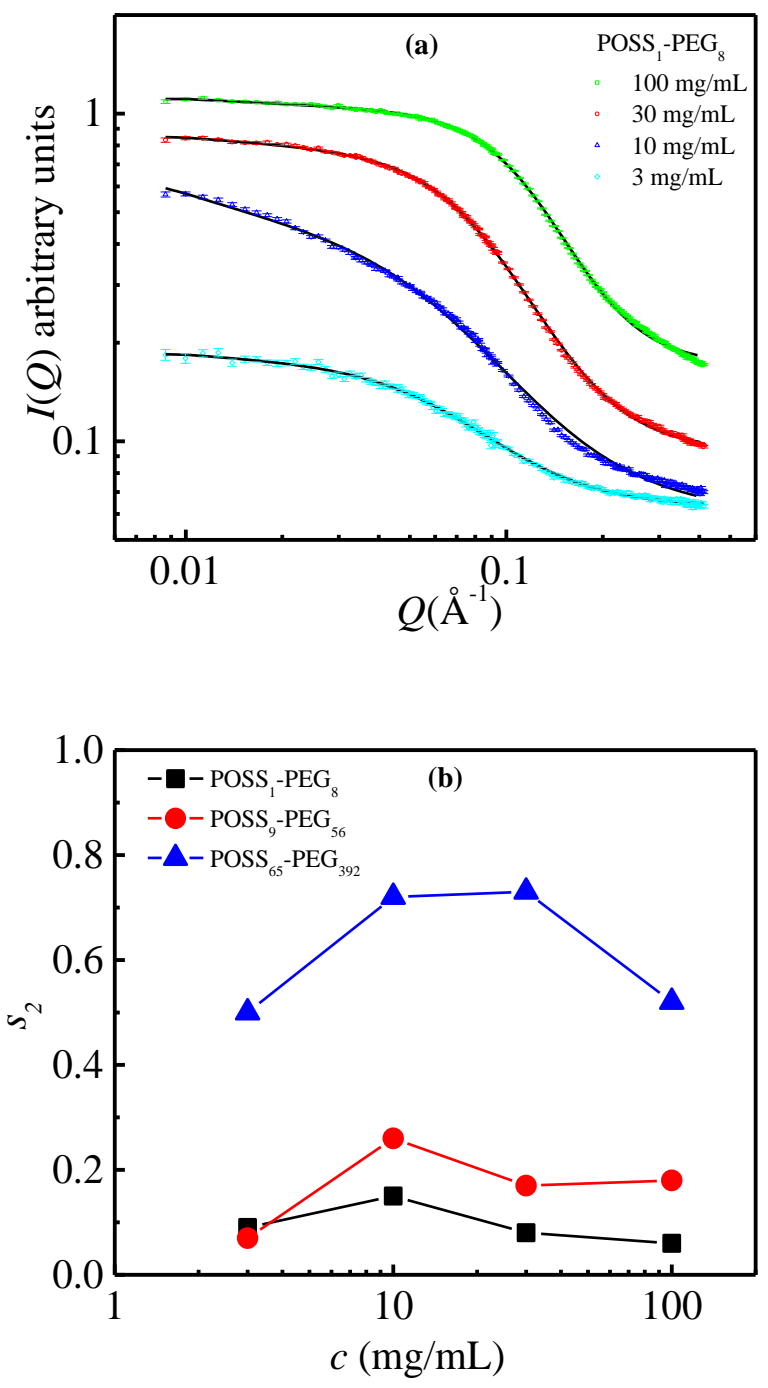

Figure 6. (a) High- $Q$ SANS signals (symbols) and the corresponding fit (black solid lines) for $\mathrm{POSS}_{1}-\mathrm{PEG}_{8}$ at four concentrations of $(100,30,10$ and 3$) \mathrm{mg} / \mathrm{mL}$. (b) Variation of elongation parameter $\mathrm{s}_{2}$ with increasing concentration for dendrimers with PEG terminal groups.

\section{Conclusions}

Six hybrid polymers containing POSS (i.e., G0, G1 and G2 dendrimers respectively with vinyl or PEG outer links) were studied by SANS. Two main scattering regimes (one with a length scale of nanometers and the other with a length scale of sub-microns) are found to contribute to the SANS signals. A new modified two correlation lengths model has been developed to analyze 
the local and global structures. The fit parameters include scale factors, correlation lengths, Porod exponents and "particle" elongation parameters. Although each specific dendrimer behaves differently to some extent in the experimental concentration range and $Q$-range, the modified two correlation lengths model quantitatively provides a reasonable description for all the data. We believe that this model could also be applied to other systems with scattering objects of two distinct sizes. For example, the two correlation lengths model could be applied to gold-nanoparticle uptaking dendrimer systems previously studied by Amis et al. ${ }^{39}$ where the indirect Fourier transformation of the scattering data showed two correlation peaks in the pair distance distribution function, and the two correlation lengths model would provide additional information including fractal and elongation parameters of the scattering objects as compared to indirect Fourier transformation results.

The most interesting part in this research is that one would have expected to observe the structure of single-dendrimer molecules at high- $Q$ and dendrimer aggregates at low- $Q$. Instead, the SANS technique uncovered an inhomogeneous morphology consisting of POSS-rich and POSS-poor regions within the dendrimers. The high- $Q$ information points to the local inhomogeneous domains. The effect of generation, terminal groups, and concentration on the local structure are discussed. It is found that at low and high mass fractions, the local structures tend to be globular, while at intermediate mass fractions, elongated structures are observed. Furthermore, it is found that PEG outer-links help to enhance the stability of inorganic POSS cages, and their flexibility allows the hybrid polymers to adjust their conformation in order to respond to the variation of external surroundings.

\section{Disclaimer / Acknowledgement}

The identification of commercial products does not imply endorsement by the National Institute of Standards and Technology nor does it imply that these are the best for the purpose. This work is based upon activities supported in part by the US National Science Foundation under Agreement No. DMR-1508249 and by the China National Science Foundation (21504096). 


\section{References}

[1] Yu X, Zhong S, Li X, Tu F, Yang S, Van Horn RM, Ni C, Pochan DJ, Quirk RP, Wesdemiotis C, Zhang WB, Cheng SZD. J Am Chem Soc 2010; 132: 16741 - 16744.

[2] Zhang W, Müller AHE. Polymer 2010; 51: 2133 - 2139.

[3] Kim BS, Mather PT. Macromolecules 2002; 35: 8378 - 8384.

[4] Zhang W, Müller AHE. Macromolecules 2010; 43: 3148 - 3152.

[5] Ye YS, Shen WC, Tseng, CY, Rick J, Huang YJ, Chang FC, Hwang BJ. Chem Commun 2011; 47: 10656 - 10658 .

[6] Maitra P, Wunder SL. Chem Mater 2002; 14: 4494 - 4497.

[7] Pyun J, Matyjaszewski K, Wu J, Kim GM, Chun SB, Mather PT. Polymer 2003; 44: $2739-2750$.

[8] Hussain H, Tan BH, Seah GL, Liu Y, He CB, Davis TP. Langmuir 2010; 26: 11763 11773.

[9] Hirai T, Leolukman M, Jin S, Goseki R, Ishida Y, Kakimoto M, Hayakawa T, Ree, M, Gopalan, P. Macromolecules 2009; 42: 8835 - 8843.

[10] Yuan H, Luo K, Lai Y, Pu Y, He B, Wang G, Wu Y, Gu ZW. Mol Pharmaceutics 2010; 7: $953-962$.

[11] Wang X, Yang Y, Gao P, Li D, Yang F, Shen H, Guo H, Xu F, Wu D. Chem Commun 2014; 50: $6126-6129$.

[12] Gnanasekaran D, Madhavan K, Beddy BSR. J Sci Ind Res India 2009; 68: 437 - 464.

[13] Cordes DB, Lickiss PD, Rataboul F. Chem Rev 2010; 110: 2081 - 2173.

[14] Kuo SW, Chang FC. Prog Polym Sci 2011; 36: 1649 - 1696.

[15] Wang F, Lu X, He CJ. Mater Chem 2011; 21: 2775 - 2782.

[16] Zhang W, Müller AHE. Prog Polym Sci 2013; 38: 1121 - 1162.

[17] Lin MC, Hsu CH, Sun HJ, Wang CL, Zhang WB, Li Y, Chen HL, Cheng SZD. Polymer 2014; $55: 4514-4520$.

[18] Kwon SJ, Kim DG, Shim J, Lee JH, Baik JH, Lee JC. Polymer 2014; 55: 2799 - 2808.

[19] Cai L, Foster C J, Liu X, Wang S, Polymer 2014; 55: 3836 - 3845.

[20] Chouwatat P, Nojima S, Higaki Y, Kojio K, Hirai T, Kotaki M, Takahara A, Polymer 2016; 84: 81 - 88 . 
[21] Tan BH, Hussain H, Lin TT, Chua YC, Leong YW, Tjiu WW, Wong PK, He CB. Langmuir 2011; 27: 10538 - 10547.

[22] Wei J, Tan BH, Bai Y, Ma J, Lu X. J Phys Chem B 2011; 115:1929 - 1935.

[23] Mya KY, Li X, Chen L, Ni X, Li J, He CB. J Phys Chem B 2005; 109: 9455 - 9462.

[24] Tan BH, Hussain H, He CB. Macromolecules 2011; 44: 622 - 631.

[25] Kuo SW, Li HF, Huang WJ, Jeong KU, Chang FC. Macromolecules 2009; 42: 1619 1626.

[26] Zhang W, Liu L, Zhuang X, Li X, Bai J, Chen Y. J Polym Sci, Part A: Polym Chem 2008; 46: $7049-7061$.

[27] Zhang W, Fang B, Walther A, Müller AHE. Macromolecules 2009; 42: 2563 - 2569.

[28] Zhang W, Yuan J, Weiss S, Ye X, Li C, Müller AHE. Macromolecules 2011; 44: 6891 6898.

[29] Zhang W, Wang S, Li X, Yuan J, Wang S. Eur Polym J 2012; 48: 720 -729.

[30] Ma L, Geng H, Song J, Li J, Chen G, Li Q. J Phys Chem B 2011; 115: 10586 - 10591.

[31] Knischka R, Dietsche F, Hanselmann R, Frey H, Mülhaupt R. Langmuir 1999; 15: 4752 -4756 .

[32] Kim KM, Keum DK, Chujo Y. Macromolecules 2003; 36: 867 - 875.

[33] Cardoen G, Coughlin BE. Macromolecules 2004; 37: 5123 - 5126.

[34] Chan ER, Zhang X, Lee CY, Neurock M, Glotzer SC. Macromolecules 2005; 38: 6168 6180.

[35] Guinier A, Fournet G. Small-Angle Scattering of X-rays. New York: John Wiley and Sons, 1955.

[36] Hammouda B. Probing nanoscale structures-The SANS toolbox. 2009. available online at http:www.ncnr.nist.gov/staff/hammouda/the sans toolbox.pdf.

[37] Mark JE, Flory PJ. J Am Chem Soc 1965; 87: 1415 - 1423.

[38] Hammouda B. J Appl Cryst 2010; 43: 716-719.

[39] Gröhn F, Bauer JB, Akpula YA, Jackson CL, Amis EJ. Macromolecules 2000; 33: 6042 -6250 . 